\title{
Right Prefrontal Cortex Responds to Item Familiarity During a Memory Encoding Task
}

\author{
P.C. Fletcher \\ Institute of Neurology, London, UK \\ R.J. Dolan
}

Institute of Neurology, and Royal Free Hospital Medical School, London, UK

\begin{abstract}
In a previous word-pair encoding study (Dolan \& Fletcher, 1997), we examined the effect of introducing novelty, either in studied words or in their mutual associations. A left medial temporal lobe (MTL) sensitivity to novel words and left prefrontal cortex (PFC) to novel associations was observed. In this further report on the data, we explored the extent to which the right PFC, more generally implicated in retrieval operations (Fletcher, Frith, \& Rugg, 1997), was sensitive to these manipulations. Specifically, we characterised changes associated with increasing familiarity of study material. We demonstrate that the response in right ventrolateral PFC is preferentially sensitive to a condition in which all material was familiar (that is, in which all material had been presented prior to scanning). A more dorsal region in right PFC was found to be relatively more active in association with a condition in which one item in the pair was familiar but was paired with a novel associate. Our results suggest that sensitivity to stimulus familiarity is expressed in right PFC, even within the context of an encoding task. The data also provide further evidence for functional heterogeneity within right PFC, with a more ventral region responding to familiarity of complete word pairs and a more dorsal region responding to familiar single words occurring in the context of new associative relationships.
\end{abstract}

\section{INTRODUCTION}

Activation of right PFC during memory retrieval is widely observed across a number of functional neuroimaging studies employing a range of psychological paradigms and test modalities (Fletcher et al., 1997; Tulving et al., 1994a). The functional significance of these observations has remained unexplained. One suggestion is that the predominance of right PFC activation during retrieval

Requests for reprints should be sent to Paul Fletcher, C. und O. Vogt Institut Für Hirnforschung, Heinrich-Hein Universitat, Universitatsstrasse 1, D 40225, Dusseldorf, Germany. Email: fletcher@hirn.uni-duesseldorf.de

PCF and RJD are supported by the Wellcome Trust. 
experiments reflects the adoption of a "retrieval mode", necessary for the initiation and maintenance of retrieval processes (Kapur et al., 1995; Nyberg et al., 1995). However, it has also been argued that right prefrontal activation is sensitive to the degree of retrieval success (Rugg et al., 1996) although it seems that retrieval occurring intentionally is associated with greater levels of right PFC activation than retrieval that is incidental to task demands (Rugg et al., 1997). It seems, therefore, that existing evidence supports the position that right PFC involvement in memory retrieval reflects both the processes involved in attempting to recall study material and those that may be contingent on the actual successful retrieval of material. Other work has suggested that right PFC shows a non-linear response to difficulty of paired associate retrieval as measured by the "semantic relatedness' of pair members (Fletcher et al., 1996). This latter response was interpreted as a reflection of post-retrieval errorchecking with the non-linearity of response reflecting at least two different types of possible error which each varied differently as a function of semantic relatedness. Other work has suggested that the region is also sensitive to processes necessary for retrieval of information regarding feature rather than location information (Nyberg et al., 1996; Owen et al., 1996).

The picture is further complicated by evidence that there is heterogeneity within right PFC with respect to sub-processes occurring in episodic memory retrieval (Fletcher et al., 1998). Tasks necessitating "monitoring" processes (Burgess \& Shallice, 1996) have been associated with activation of dorsal right PFC whereas simpler, externally specified retrieval processes, not necessitating monitoring, are associated with activation of more ventral PFC.

Clearly, our understanding of the significance of right PFC activation in association with memory retrieval is incomplete with respect to the processes subserved and to the functional heterogeneity within PFC. The current experiment was designed to explore brain systems associated with the encoding of word paired associates and has already been reported as such (Dolan \& Fletcher, 1997). However, the basic study design, which characterised the effects of novelty, may also produce interesting effects with respect to growing familiarity of study material and the possibility of this engendering retrieval, whether incidental or intentional, even though such retrieval would be occurring in the face of an encoding task. In this treatment of the data, we examine effects associated with this familiarity. During positron emission tomography (PET) scanning, subjects were presented with lists of word pairs, each pair consisting of a category and an exemplar. These pairs were, in the context of the experiment, novel or familiar with respect to both the words themselves and the semantic linkages between them. We showed that left dorsolateral prefrontal cortex (DLPFC) was sensitive to a manipulation of the association between category and exemplar, that is, maximal activation in this region was seen in scans involving a change in category-examplar pairings. By contrast the medial temporal cortex, including the hippocampus and parahippocampal region, showed a response that 
was maximal when the entire word pair was novel. This analysis of the data has been reported and discussed elsewhere (Dolan \& Fletcher, 1997). The present analysis of the data thus involves a reversal of our previous analyses. In simple terms, instead of characterising brain changes occurring in association with novel compared to familiar stimuli, we now explored changes occurring in association with familiar compared to novel stimuli. This extension of our original analysis was motivated by post hoc reports from all subjects that they frequently, and spontaneously, recalled previous presentations of familiar items in response to cueing even though the experimental task did not explicitly require this. This observation is of particular interest in the light of the aforementioned confusion regarding the role of right $\mathrm{PFC}$ in episodic memory retrieval and the array of functional neuroimaging studies that have shown this region to be involved in many different retrieval situations (Buckner et al., 1996; Fletcher et al., 1996, 1998; Kapur et al., 1995; Nyberg et al., 1996; Rugg et al., 1996; Shallice et al., 1994; Tulving et al., 1994a,b; Wheeler, Stuss, \& Tulving, 1997). Most previous studies have examined retrieval-related brain systems in the context of tasks that have explicitly required subjects to recall previously presented material (Fletcher et al., 1997). In the current study, any retrieval was incidental to the task demands and, thus, provides a different setting in which to explore retrieval-related brain responses.

\section{METHOD}

\section{PET Scanning}

Six healthy male right-handed volunteers were studied using a SIEMENS/CPS ECAT EXACT HR+ (MODEL 962) PET scanner in 3-D mode with a $15 \mathrm{~cm}$ axial field of view. Relative $\mathrm{rCBF}$ was measured from the distribution of radioactivity after slow bolus i.v. injection of $\mathrm{H}_{2}{ }^{15} \mathrm{O}(9 \mathrm{mCi}$ per scan, each lasting 90 seconds). Attenuation-corrected data were reconstructed into 63 image planes with a resulting resolution of $6 \mathrm{~mm}$ at full-width-half-maximum. For each subject, structural magnetic resonance (MR) images were obtained with a 2 T Magnetom VISION (Siemens, Germany).

\section{Psychological Tasks}

Prior to each PET scan, subjects were presented verbally with a list of categoryexemplar word pairs (e.g. DOG...BOXER). The list was presented twice, during a 90 -second lead-in period, with a third presentation timed to coincide with the onset of PET scanning. Subjects were instructed to try to remember material for later testing. They were unaware of when scanning was actually occurring, in order to ensure, as far as possible, that they attended to each of the list presentations. During the third presentation (that is, during scanning), one of the following manipulations was made: 
1. The same list was presented for the third time. That is, all material was familiar. We shall henceforth refer to this condition as the Wholly Familiar condition.

2. For each pair, either the category or the exemplar was changed. (Thus, for example, DOG...BOXER might become DOG...LABRADOR or SPORTSMAN...BOXER). In this case, material was partly familiar. Although the [New Category...Old exemplar and [Old Category...New Exemplar] conditions were actually scanned separately, we have collapsed them for the purposes of the current analysis and would point out, with respect to the prefrontal regions discussed, that there was no difference in levels of activity between them. We shall refer to this condition as the Partially Familiar condition.

3. An entirely new list of word pairs was presented. We shall refer to this as the Wholly Novel condition.

The order of the presentation of experimental conditions was counterbalanced both within and across subjects.

\section{Data Analysis}

Statistical parametric mapping (SPM96) software was used for image realignment, transformation into standard stereotactic space, smoothing, and statistical analysis (Friston et al., 1995). All measurements per condition were averaged across subjects. State-dependent differences in global flow were covaried out using ANCOVA. Main effects and interactions were assessed with contrasts of the adjusted task means using $t$-statistic subsequently transformed into normally distributed $Z$ statistic. The resulting set of $Z$ values constituted a statistical parametric map $\left[\mathrm{SPM}_{(z)}\right]$ which was then thresholded at $P<.001$. The following comparisons were made. Scans in which presented material was wholly familiar and those in which material was partly familiar were compared separately with those in which material was completely novel. A further comparison was made directly between the wholly and partially familiar conditions.

\section{RESULTS}

\section{Behavioural Results}

The effectiveness of encoding was assessed using a cued retrieval task after a five-minute interval. These data showed recall was $95 \%$ for the wholly novel condition, $78 \%$ for the partially familiar condition, and $93 \%$ for the wholly familiar condition. It should be noted that the significantly lower level of recall with respect to the partially familiar condition reflects proactive interference and has been discussed elsewhere (Dolan \& Fletcher, 1997). 


\section{Neuroimaging Results}

Unless otherwise stated, the statistical parametric maps were thresholded at $P<.001$, uncorrected for multiple comparisons.

Wholly Familiar vs Wholly Novel. Wholly familiar stimuli were associated with relatively greater activation in bilateral anterior PFC, in dorsal and ventral regions of the right middle frontal gyrus and in medial and lateral parietal cortex (see Table 1 and Plate 3).

Partially Familiar vs Wholly Novel. Partially familiar stimuli were associated with relatively greater activation in bilateral anterior PFC, in a dorsal region of the right middle frontal gyrus and in medial and lateral parietal cortex (see Table 2 and Plate 4).

TABLE 1

Regions Showing Significantly Greater Activity in Association with the Presentation of Wholly Familiar Compared to Wholly Novel Material

\begin{tabular}{llc}
\hline Region & Coordinates* $^{*}$ & Z Score \\
\hline R. Middle frontal gyrus (BA 10) & $40,56,-4$ & 4.5 \\
L. Middle frontal gyrus (BA 10) & $-32,58,0$ & 4.1 \\
R. Inferior frontal gyrus (BA 44/45) & $48,12,12$ & 3.3 \\
R. Middle frontal gyrus (B A 9/46) & $44,24,30$ & 3.3 \\
Medial parietal cortex (BA 7) & $-2,-74,52$ & 3.3 \\
\hline
\end{tabular}

*Talairach and Tournoux, 1988.

TABLE 2

Regions Showing Significantly Greater Activity in Association with the Presentation of Partially Familiar Compared to Wholly Novel Material

\begin{tabular}{llc}
\hline Region & Coordinates* $^{*}$ & Z Score \\
\hline R. Middle frontal gyrus (BA 10) & $30,58,-2$ & 4.1 \\
L. Middle frontal gyrus (BA 10) & $-32,58,0$ & 5.5 \\
R. Middle frontal gyrus (B A 9/46) & $44,20,26$ & 4.0 \\
& $42,8,36$ & 3.2 \\
L. Middle frontal gyrus (BA 9) & $-46,20,40$ & 4.9 \\
Medial parietal cortex (BA 7) & $0,-70,48$ & 6.2 \\
R. Lateral parietal cortex (BA 7) & $28,-54,50$ & 4.2 \\
L. Lateral parietal cortex (BA 7) & $-36,-54,48$ & 4.2 \\
\hline
\end{tabular}

*Talairach and Tournoux, 1988. 
Wholly familiar vs Partial Familiar. Compared to the partial familiar condition, the wholly familiar condition was associated with relatively greater levels of activity in bilateral anterior temporal regions and right medial parietal cortex.

Partially Familiar vs Wholly Familiar. This comparison showed the partially familiar condition to be associated with greater activity in left dorsolateral prefrontal cortex, left medial and lateral parietal cortex.

In view of the particular interest in right PFC with respect to item familiarity, we repeated the last two contrasts at a reduced level of significance $(P<.05$, uncorrected), concentrating solely on the dorsal and ventral right PFC which had been shown to be activated in the two familiar conditions (wholly familiar and partially familiar) compared to the wholly novel condition. These comparisons showed a dissociation in the ventral and dorsal regions with greater levels of activity in ventral right $\mathrm{PFC}$ in the wholly compared to the partially familiar condition, and greater levels of activity in the dorsal right PFC in the reverse contrasts. These results are summarised in Plates 3 and 4.

\section{DISCUSSION}

Our data demonstrate that right PFC is sensitive to the degree of stimulus familiarity and that this sensitivity can be seen even in a task designed to engage encoding processes. Moreover, there was subtle evidence of a dissociation in the observed patterns of activity occurring in dorsal and ventral right PFC. The latter showed an apparently linear relationship with the degree of stimulus familiarity. Activity here was maximal when all pair members presented during the scan had already been presented twice during the lead-in period. The more dorsal region, on the other hand, was maximally sensitive to the condition in which subjects had become familiarised with only part of the stimuli (i.e. one item in each pair).

Before discussing these results in greater detail, it is important to raise a number of caveats. Primarily, the experiment was designed to look at the effects of stimulus novelty during memory encoding and the condition that has been considered as the "activation" task in the current treatment of the data was originally used as the "baseline". The current analysis is reported because of the interesting findings with respect to right PFC but we are suitably cautious about drawing firm conclusions over an issue that the experiment was not designed to address. In addition, the subtlety of the findings (most particularly with reference to the ventral-dorsal dissociation, which only survived a lenient statistical threshold for significance) is another reason for caution. Nevertheless, we believe that the results bear further discussion for a number of reasons. First, it is both interesting and potentially informative that the left and right prefrontal regions are responding to task demands with highly different qualitative patterns 
of response. Second, it is a reminder that task manipulations may produce effects associated with processes beyond those that the tasks are explicitly designed to engage, even though the task may be designated and considered as a fairly lowlevel baseline condition. Finally, the observation of different patterns of familiarity response in ventral and dorsal regions of right PFC is worth speculating upon.

Overall, the right PFC region showing a response to familiarity encompassed the ventral areas of the inferior frontal gyrus, bordering upon and, perhaps, extending into the insula, and a more dorsal region of the inferior frontal sulcus. In previous studies, activation of right PFC has been found in association with memory retrieval (Fletcher et al., 1997; Fletcher et al., 1998; Shallice et al., 1994; Squire et al., 1992; Tulving et al., 1994b). The finding has been framed in terms of the adoption of a retrieval mode or of processes subserving an active search of memory contents. However, our data suggest that right prefrontal activation can occur in association with the presentation of previously learned material even in the context of a task in which these processes are not explicitly operative. Rather, it is possible that incidental retrieval will also engage right prefrontally mediated processes. An important question here is whether subjects were actually retrieving material incidentally. Incidental retrieval can refer to retrieval that is incidental to task demands (i.e. not required to perform the allotted task) or retrieval that occurs incidentally without subjects engaging in an effortful memory search (that is, incidental as opposed to intentional retrieval). With respect to the first definition, we can be confident that the retrieval was incidental. However, with respect to the second and more interesting definition, the case is less clear cut. Although subjects reported that, in the Wholly Familiar condition, presentation of one item was often associated with effortless retrieval of its pair, it is nevertheless possible that the right PFC activation actually reflects a more effortful process. Moreover, recent findings have suggested that right PFC (in a dorsal region close to the one reported here) shows higher levels of activity in intentional compared to incidental retrieval (Rugg et al., 1997). Aside from post hoc subjects' reports, we have no clear way of addressing the question of to what extent subjects' retrieval was truly incidental. Nevertheless, the different patterns of activity observed in the dorsal and ventral foci of activation may offer some clues as will be discussed next.

One possibility, concerning the functional significance of our findings, is that the activations simply reflect the recognition, during scanning, of items that had been presented during the lead-in period. This would be consistent with a previous study of word recognition memory (Rugg et al., 1996) where word lists containing a higher density of previously presented items were associated with activation of right PFC when compared to lists consisting entirely of previously unseen items. However, it should also be noted that other experiments with similar designs have concluded that the right PFC activation reflects retrieval effort rather than the actual recognition of items (Kapur et al., 1995; Nyberg et 
al., 1995). A related but alternative possibility is that activation of right PFC reflects automatic item retrieval in response to verbal cueing. Thus, for example, when a word pair such as DOG...BOXER was presented for the third time (the third presentation occurring during scanning), the presentation of the category DOG results in automatic retrieval of the exemplar BOXER. Indeed subjects reported, at debriefing, that this was so in the Wholly Familiar condition, remarking that presentation of the categories resulted in the automatic retrieval of the appropriate exemplar, pre-empting its presentation by the experimenter. Thus, right PFC activity may reflect recognition or cued retrieval of paired associates. If it reflects cued retrieval then, as remarked earlier, it is not entirely clear whether this is incidental or intentional.

However, the observed functional heterogeneity within right PFC is interesting and possibly informative with respect to this uncertainty. The more ventral region showed a greater sensitivity to the Wholly Familiar condition, whereas the more dorsal region showed greater sensitivity to the Partially Familiar condition. This observation of a dissociation between more dorsal and ventral regions is in keeping with previous functional neuroimaging experiments of episodic memory retrieval (Fletcher et al., 1998) and with evidence from monkey experiments which have suggested that, in working memory tasks, dorsal and ventral regions of PFC subserve qualitatively different processes (Petrides, 1994, 1995). Moreover, it suggests that the two regions subserve qualitatively different processing.

Regarding the more ventral right PFC activation, it was preferentially sensitive to lists in which items were wholly familiar. Recall that this was the condition in which subjects reported incidental retrieval occurring in anticipation of the experimenter's presentation of the exemplar. By contrast, in the condition where material was only partly familiar, they reported that they tended to do this less as it was unhelpful to the experimental task-the task instructions being to encode the new category-exemplar pairings (although subjects were not made aware of when scanning was occurring, nor were they informed as to the nature of the experimental manipulations, the blocked presentations meant that they nevertheless realised the nature of changes and that these changes occurred during the third presentation of a list). This suggestion, that the more ventral activation reflects automatic retrieval of exemplars in response to category presentation, is in keeping with a previous study (Fletcher et al., 1998) showing that right ventral PFC is most active with cued paired associate retrieval. Our interpretation was that the activation in this region reflected retrieval specification (as determined by each successive category cue) across the course of the scan. The present finding is consistent with this interpretation in regard to the Wholly Familiar condition. On the other hand, the absence of ventral PFC activation in the Partially Familiar condition (at the pre-set threshold for statistical significance) is consistent with the subjective reports of participants that automatic retrieval of paired associates was unhelpful to the experimental 
task in this condition. However, it should be noted that right ventral PFC activity in this condition was intermediate between the Wholly Familiar and Wholly Novel conditions and, at a lower threshold for significance $(P<.01$, uncorrected $)$ activation was seen here in association with the Partially Familiar condition. In this condition, the familiarity of the presented categories may have led to some spontaneous recovery of their previous associates but, as subjects found this unhelpful, there may have been an active suppression of this phenomenon. Alternatively, it might simply be the case that this region was responsive purely to the amount of familiar material within a scanning block. That is, it is possible that every time subjects recognised an item that had been previously presented, then activation occurred irrespective of whether or not presentation of that item provoked cued retrieval of its previously learned associate.

With respect to the pattern of activity seen in the more dorsal region of right PFC, these two possible interpretations are less plausible. Activity here was greater in both the Wholly and the Partially Familiar conditions when compared separately with the Wholly Novel condition. A direct comparison of the Wholly and the Partially Familiar conditions at a reduced threshold for significance $(P<.05$, uncorrected) showed a relatively greater activation of the dorsal region in response to the latter condition. This is a complex finding which cannot be attributed simply to stimulus familiarity, as activity was maximal in the condition where only half of the material was familiar. It also seems unlikely that the observed pattern of activity in this region reflects automatic cued retrieval, as the extent to which such retrieval was occurring was maximal in the Wholly Familiar condition. Rather, we suggest that activity here reflects a more active processing. More specifically, if the activation in this region reflected purely retrieval-related processing (whether recognition, incidental, or intentional cued retrieval, as discussed earlier) then it would be maximal in the Wholly Familiar condition. The Partially Familiar condition, in which it achieved peak activation, is one in which these forms of automatic retrieval would be unhelpful, and, perhaps, a hindrance. We suggest, therefore, that the dorsal right PFC activation might reflect processes that check/monitor (Burgess $\&$ Shallice, 1996) the products of this unnecessary and unhelpful retrieval. This, of course, is highly speculative but it is noteworthy that, in a recent study (Fletcher et al., 1998), we demonstrated greater activation of right dorsal PFC in a retrieval task that required monitoring of retrieval products. In the case of the Partially Familiar condition, such monitoring would, we suggest, be engaged to a greater extent than in the Wholly Familiar condition. In the Partially Familiar condition, word pairs each contained one new item and, therefore, a new associative relationship. Thus, in this condition, the previously learned association to each item would need to be suppressed or adjusted. In our previous reporting of these data (Dolan \& Fletcher, 1997), we noted that this condition was most prominently associated with activation of left PFC and we interpreted this observation in terms of proactive interference or the active 


\section{FLETCHER AND DOLAN}

formation of new semantic associations to stimuli that had been previously presented with different associations. It seems plausible that this condition also engages right dorsal PFC to a lesser extent (which did not survive our previously more stringent statistical threshold), and that left and right prefrontal cortices act in conjunction, the latter involved in the retrieval and monitoring of previously learned associations and the former engaged in the formation of the new ones and, perhaps, suppression of the previously learned responses.

In summary, these data indicate that right PFC activation associated with explicit memory retrieval may occur in the absence of an experimental requirement to retrieve material. That is, certain processes, subserved by right prefrontal function may be engaged automatically and/or incidentally when familiar items are presented. Further, the precise regions of prefrontal cortex activated are anatomically distinct, and dependent on the nature of these processes and the extent to which they are appropriate to the context of the experiment. Although these findings must be treated with all due caution, arising as they do from a post hoc analysis of data, we suggest that they may nevertheless be informative with respect to the frequently reported activation of right $\mathrm{PFC}$ in association with memory retrieval.

\section{REFERENCES}

Buckner, R.L., Raichle, M.E., Miezin, F.M., \& Petersen, S.E. (1996). Functional anatomic studies of memory retrieval for auditory words and visual pictures. Journal of Neuroscience, 16(19), 6219-6235.

Burgess, P.W., \& Shallice, T. (1996). Confabulation and the control of recollection. Memory, 4(4), 359.

Dolan, R.J., \& Fletcher, P.C. (1997). Dissociating prefrontal and hippocampal function in episodic memory encoding. Nature, 388, 582-585.

Fletcher, P.C., Frith, C.D., \& Rugg, M.D. (1997). The functional neuroanatomy of episodic memory. Trends in Neurosciences, 20(5), 213-218.

Fletcher, P.C., Shallice, T., Frith, C.D., Frackowiak, R.S.J., \& Dolan, R.J. (1996). Brain activity during memory retrieval: The influence of imagery and semantic cueing. Brain, 119, 1587-1596.

Fletcher, P.C., Shallice, T., Frith, C.D., Frackowiak, R.S.J., \& Dolan, R.J. (1998). The functional roles of the prefrontal cortex in episodic memory: II Retrieval. Brain, 121, 1249-1256.

Friston, K.J., Holmes, A.P., Worsley, K.J., Poline, J.B., Frith, C.D., \& Frackowiak, R.S.J. (1995). Statistical parametric maps in functional imaging; a general linear approach. Human Brain Mapping, 2, 189-210.

Kapur, S., Craik, F., Brown, G.M., Houle, S., \& Tulving, E. (1995). Functional role of the prefrontal cortex in memory retrieval: A PET study. NeuroReport, 6, 1880-1894.

Nyberg, L., McIntosh, A.R., Cabeza, R., Habib, R., Houle, S., \& Tulving, E. (1996). General and specific brain regions involved in encoding and retrieval of events: What, where and when. Proceedings of the National Academy of Science, USA, 93, 11280-11285.

Nyberg, L., Tulving, E., Habib, R., Nilsson, L.G., Kapur, S., Cabeza, R., \& McIntosh, A.R. (1995). Functional brain maps of retrieval mode and recovery of episodic information. NeuroReport, 7 , 249-252.

Owen, A.M., Milner, B., Petrides, M., \& Evans, A.C. (1996). Memory for object features versus memory for object location: A positron emission tomography study of encoding and retrieval processes. Proceedings of the National Academy of Science, USA, 93, 9212-9217. 
Petrides, M. (1994). Frontal lobes and working memory: Evidence from investigations of the effects of cortical excisions in non-human primates. In F. Boller \& J. Grafman (Eds.), Handbook of neuropsychology (Vol. 9) (pp.59-82). Amsterdam: Elsevier Science.

Petrides, M. (1995). Impairments on non-spatial self-ordered working memory tasks after lesions to the mid-dorsal part of the lateral frontal cortex in the monkey. Journal of Neuroscience, 15(1), $359-375$.

Rugg, M.D., Fletcher, P.C., Frith, C.D., Frackowiak, R.S.J., \& Dolan, R.J. (1996). Differential activation of the prefrontal cortex in successful and unsuccessful memory retrieval. Brain, 119, 2073-2083.

Rugg, M.D., Fletcher, P.C., Frith, C.D., Frackowiak, R.S.J., \& Dolan, R.J. (1997). Brain regions supporting intentional and incidental memory: A PET study. NeuroReport, 8(5), 1283-1287.

Shallice, T., Fletcher, P., Frith, C.D., Grasby, P., Frackowiak, R.S.J., \& Dolan, R.J. (1994). Brain regions associated with acquisition and retrieval of verbal episodic memory. Nature, 368, 633635.

Squire, L.R., Ojemann, J.G., Miezin, F.M., Petersen, S.E., Videen, T.O., \& Raichle, M.E. (1992). Activation of the hippocampus in normal humans: A functional anatomical study of memory. Proceedings of the National Academy of Science, USA, 89, 1837-1841.

Talairach, J., \& Tournoux, P. (1988). Co-planar stereotaxic atlas of the human brain. Stuttgart: George Theme Verlag.

Tulving, E., Kapur, S., Craik, F.I.M., Moscovitch, M., \& Houle, S. (1994a). Hemispheric encoding/ retrieval asymmetry in episodic memory: Positron emission tomography findings. Proceedings of the National Academy of Science, USA, 91, 2016-2020.

Tulving, E., Kapur, S., Markovitsch, H.J., Craik, F.I.M., Habib, R., \& Houle, S. (1994b). Neuroanatomical correlates of retrieval in episodic memory: Auditory sentence recognition. Proceedings of the National Academy of Science, USA, 91, 2012-2015.

Wheeler, M.A., Stuss, D.T., \& Tulving, E. (1997). Towards a theory of episodic memory: The frontal lobes and autonoetic consciousness. Psychological Bulletin, 121(3), 331-354. 
sagittal

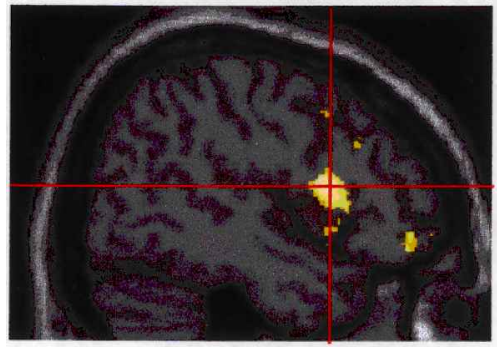

\section{transverse}

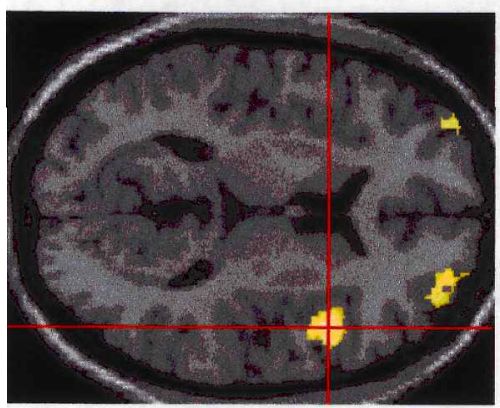

coronal
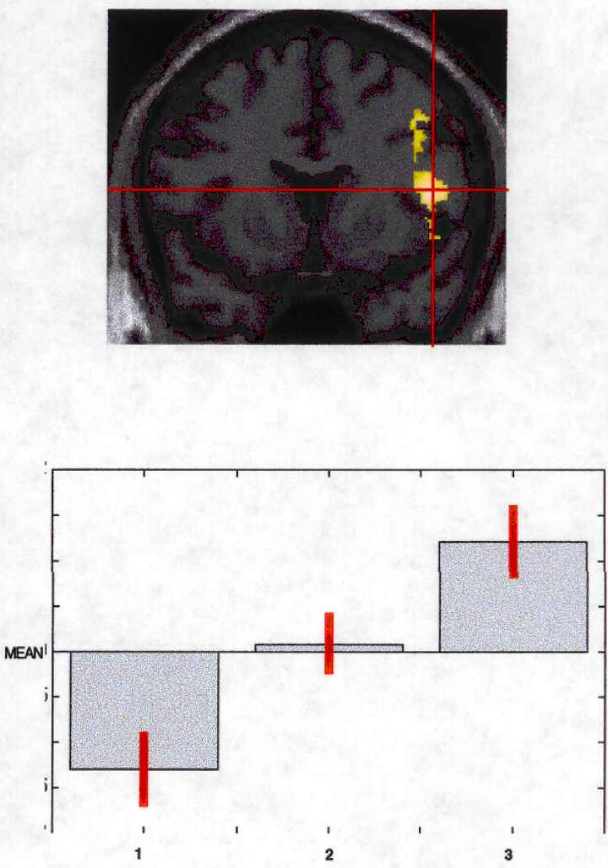

PLATE 3. Regions of PFC showing a significant activity in association with the presentation of wholly familiar compared to wholly novel material. A statistical parametric map rendered onto sections of a stereotactically normalised structural mri is shown. The sections were chosen (coordinates [Talairach \& Tournoux, 1988] X, Y, Z $=48,12,12$ ) to show area of prefrontal activation. In the bottom right panel is shown the plot of parameter estimates for this voxel $(48,12$, 12) for conditions 1 (wholly novel material), 2 (partially familiar), and 3 (wholly familiar). It can be seen that the level of activity in the region is highest in association with the presentation of wholly familiar material, with the partially familiar condition showing an intermediate level of activity. 


\section{sagittal}

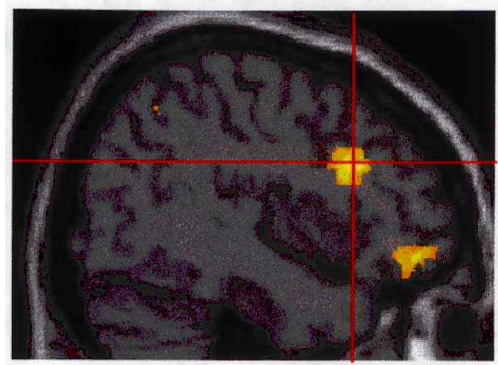

coronal

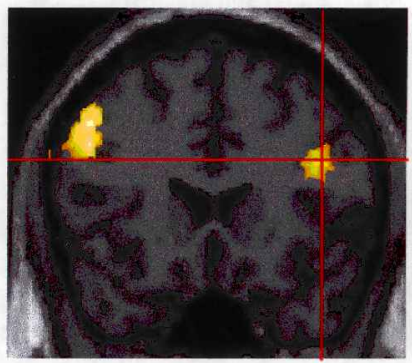

\section{transverse}
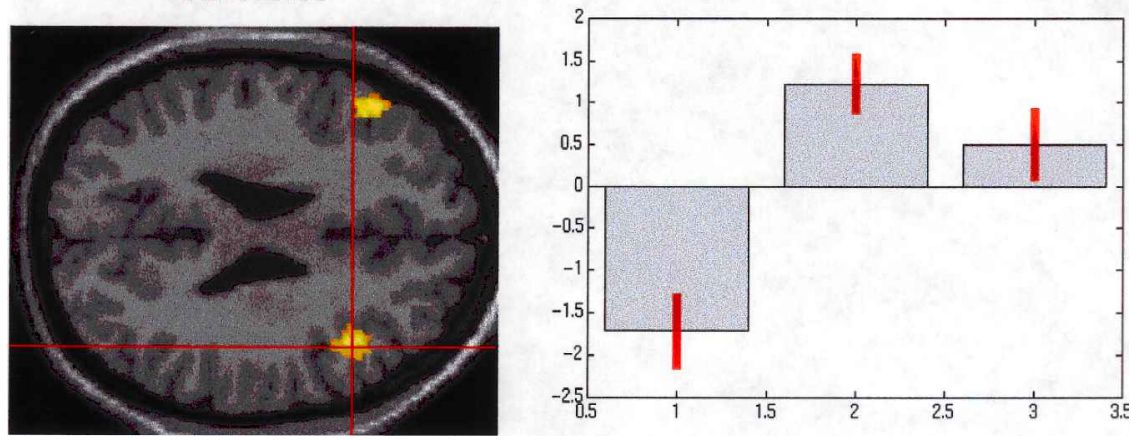

PLATE 4. Regions of PFC showing a significant activity in association with the presentation of partially familiar compared to wholly novel material. A statistical parametric map rendered onto sections of a stereotactically normalised structural $\mathrm{mri}$ is shown. The sections were chosen (coordinates [Talairach \& Tournoux, 1988] X, Y, Z =44, 20, 26) show a more dorsal region of right PFC. In the bottom right panel is shown the plot of parameter estimates for this voxel for conditions 1 (wholly novel material), 2 (partially familiar), and 3 (wholly familiar). It can be seen that the level of activity in the region is highest in association with the presentation of partially familiar material, with the wholly familiar condition reflected in an intermediate level of activity. 